\title{
Oscillation Regularity in Noise-Driven Excitable Systems with Multi-Time-Scale Adaptation
}

\author{
William H. Nesse \\ University of Utah \\ Christopher A. Del Negro \\ College of William and Mary, cadeln@wm.edu
}

Paul C. Bressloff

University of Utah

Follow this and additional works at: https://scholarworks.wm.edu/aspubs

Part of the Applied Mathematics Commons, and the Biology Commons

\section{Recommended Citation}

Nesse, William H.; Del Negro, Christopher A.; and Bressloff, Paul C., Oscillation Regularity in Noise-Driven Excitable Systems with Multi-Time-Scale Adaptation (2008). Phys Rev Lett, 101(8).

https://doi.org/10.1103/PhysRevLett.101.088101

This Article is brought to you for free and open access by the Arts and Sciences at W\&M ScholarWorks. It has been accepted for inclusion in Arts \& Sciences Articles by an authorized administrator of W\&M ScholarWorks. For more information, please contact scholarworks@wm.edu. 


\title{
Oscillation Regularity in Noise-Driven Excitable Systems with Multi-Time-Scale Adaptation
}

\author{
William H. Nesse, ${ }^{1}$ Christopher A. Del Negro, ${ }^{2}$ and Paul C. Bressloff ${ }^{1}$ \\ ${ }^{1}$ Department of Mathematics, University of Utah, Salt Lake City, Utah 84112, USA \\ ${ }^{2}$ Department of Applied Science, College of William and Mary, Williamsburg, Virginia 23187, USA
}

(Received 17 January 2008; published 20 August 2008)

\begin{abstract}
We investigate oscillation regularity of a noise-driven system modeled with a slow afterhyperpolarizing adaptation current (AHP) composed of multiple-exponential relaxation time scales. Sufficiently separated slow and fast AHP time scales (biphasic decay) cause a peak in oscillation irregularity for intermediate input currents $I$, with relatively regular oscillations for small and large currents. An analytic formulation of the system as a stochastic escape problem establishes that the phenomena is distinct from standard forms of coherence resonance. Our results explain data on the oscillation regularity of the pre-Bötzinger complex, a neural oscillator responsible for inspiratory breathing rhythm generation in mammals.
\end{abstract}

DOI: 10.1103/PhysRevLett.101.088101

PACS numbers: 87.19.1c, 87.19.1n

The oscillation period of a neural dynamical system typically depends on the relaxation time scales of one or more adaptation processes that terminate the duty cycle. The particular duty cycle of the neural system could be a spike or burst of spikes in a single neuron, or an oscillation in the average firing of a population of neurons. It is common that the relaxation of these adaptation mechanisms is modeled in terms of an outward afterhyperpolarization (AHP) current with a single exponential decay to a reexcitation threshold. An important observation of single-time-scale noise-driven systems is that the oscillation regularity, as measured by the coefficient of variation (CV) of the periods (standard deviation over the average of the period), can exhibit nonmonotonic behavior as a function of noise strength (e.g., coherence resonance [1]), but exhibit monotonic increase in regularity (decrease of CV) as a function of excitability [2]. However, it is common that neural systems possess more than one adaptation mechanism spanning a range of time scales [3]. An important issue that follows from this is how the regularity of oscillations in noise-driven systems are affected by multi-time-scale adaptation.

In this Letter we investigate what impact the interaction of white noise inputs and multi-time-scale adaptation processes have on the regularity of oscillations in neural systems, and then apply these results to the neural oscillator involved in generating the mammalian breathing rhythm. In contrast to single exponential time-scale systems, we find that if the multiple AHP relaxation time scales are sufficiently separated so as to exhibit a biphasic decay composed of a relatively fast decay followed by a relatively slow decay, then the system will exhibit a nonmonotonic peak in the $\mathrm{CV}$ for intermediate excitability, set by an input current. We show that this phenomenon holds for a wide range of noise strengths, and is distinct from standard forms of coherence and anticoherence resonance $[1,2]$. In addition, we show how the phenomenon can be understood in terms of a simple stochastic escape problem from a slowly varying potential well governing noisedriven motion to a threshold. Finally, our results provide a novel explanation for experimental data regarding oscillation regularity in the pre-Bötzinger complex (pre-BötC) isolated in a rhythmically active mammalian in vitro slice. The pre-BötC is the kernel for the mammalian inspiratory breathing rhythm [4], with periods from 2-3 min to $2 \mathrm{sec}$, suggesting that a wide range of adaptation time scales may be involved. These oscillations have recently been modeled as a noise-driven recurrent excitatory neural network whose dynamics can be captured by a low-dimensional mean-field model similar to that studied below [5].

Consider a simple cubic FitzHugh-Nagumo-like model for the activity $z$ of an oscillatory system:

$$
\dot{z}=-z(z+1)(z-1)-H+I+\sigma \xi(t),
$$

where $\xi(t)$ is an uncorrelated Gaussian process: $\langle\xi(t)\rangle=0$, $\left\langle\xi(t) \xi\left(t^{\prime}\right)\right\rangle=\delta_{t, t^{\prime}}, I$ is the input current, and $\sigma$ is the noise strength. The variable $H=\sum_{j=1}^{M} h_{j}$ is the sum of AHP currents $h_{j}$, each governed by the equation

$$
\tau_{j}(z) \dot{h}_{j}=-h_{j}+a_{j} g(z), \quad j=1 \ldots M,
$$

where $\tau_{j}(z)=\tau_{j}+\left(\tau_{\text {up }}-\tau_{j}\right) g(z)$, and $\tau_{j}$ are the relaxation time constants, $g(z)=1 /(\exp [-80(z-0.5)]+1) \in$ $(0,1)$ is a sigmoidal activation curve with threshold 0.5 and gain 80 (which approximates a Heaviside function), and $\tau_{u p}=2 \mathrm{msec}$ is the activation time constant for each AHP current: when the system is in the active phase $(z>0.5)$, each of the AHP currents activates toward their respective $a_{j}$ activation strengths at the same rate $1 / \tau_{u p}$, but after activity termination $(z<0.5)$, each AHP relaxes back toward zero with time constants $\tau_{j}$, respectively. Our AHP model is simple but the underlying mechanisms can be diverse [3], from fast voltage-gating (millisecond time scales), slower second-messenger activation (subsecond 
time scales), to much slower regulatory phosphorylation and ion channel insertion (second and multisecond time scales). The cubic function in (1) has been employed to model spike activity as in the FitzHugh-Nagumo model [1] as well as approximate mean-field models of populationlevel activity [5]. With zero noise, $z$ and $H$ settle to a low activity steady state for $I<0.4$. At $I \sim 0.4$ the steady state disappears and forms an oscillation through a saddle node on an invariant cycle (SNIC) bifurcation. The oscillations also terminate for $I$ above a ceiling threshold; however we do not investigate oscillations near this upper threshold.

First, keeping noise strength constant $(\sigma=0.1)$, we perform Monte Carlo simulations to compute the CV and the period as a function of the input $I$ and the AHP timescale composition. Figure 1 left column [panels (a)-(c)] shows the mean frequency $1 /\langle T\rangle$, standard deviation of the periods $\sigma_{T}$, and the $\mathrm{CV}$, respectively, comparing a singletime-scale AHP system $\tau_{1}=10 \mathrm{msec}\left(a_{1}=2\right.$, solid black dots), with four two-time-scale systems possessing both the short time-scale $\tau_{1}$ at $a_{1}=1.5$ strength with $a_{2}=0.5$ strength (note $a_{1}+a_{2}=2$ ) of varying longer time-scale separations $\tau_{2}=100,500,1000$, and $5000 \mathrm{msec}$, indicated by ball-line, dashed, dotted, dot-dashed, and solid lines, respectively. Each graphed point represents 400 oscillations. The right column of Fig. 1 shows the same results computed via the analytic stochastic escape formulation which is derived later. Decreasing the excitability below the deterministic threshold for oscillations $(I \sim 0.4)$, indicated by the vertical dashed line, reduces the frequency to near zero (a) while the $\sigma_{T}$ (b) diverges, and the CV
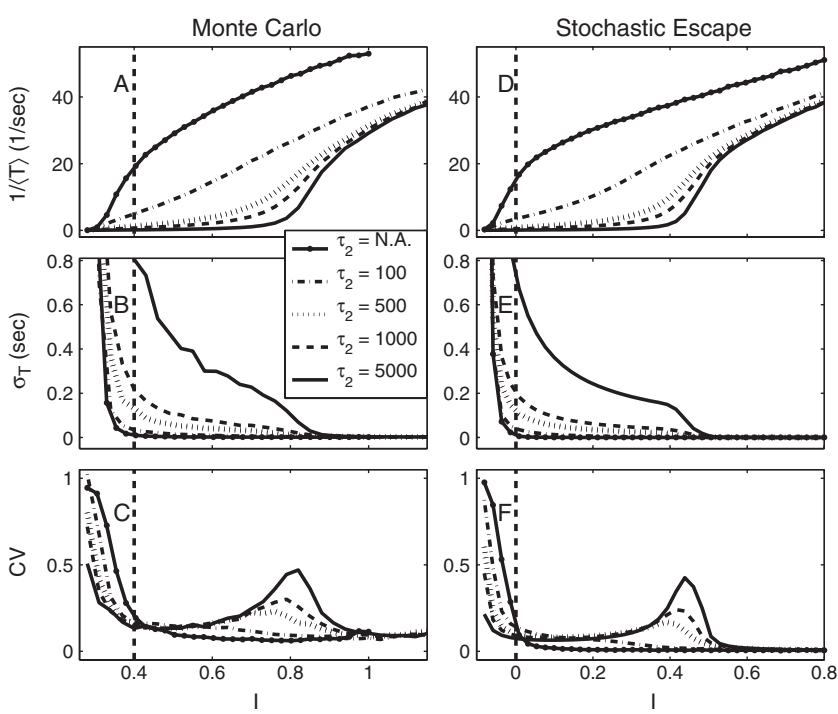

FIG. 1. Five AHP currents for Monte Carlo simulations (a)-(c) and the stochastic escape formulation (d)-(f). The mean firing rate $1 /\langle T\rangle$, SD of the period $\sigma_{T}$, and $\mathrm{CV}$, are shown for range of inputs $I$. The vertical dashed line indicates deterministic threshold of oscillation. The single-time-scale model $\left(\tau_{1}=10 \mathrm{msec}\right)$ is compared with four two-time-scale models [panel (b) legend]: the wider the two-timecale separation, the greater the peak in the CV. (c) approaches unity at varying rates for each model. This below-threshold behavior is standard for all noisedriven systems regardless of AHP dynamics. However, increasing excitability above threshold reveals heterogeneous behavior: The single-time-scale system exhibits a monotonically decreasing then flat $I-\mathrm{CV}$ relationship while the two-time-scale models produce large nonmonotonic, peaked $I-C V$ relationships, where the peak amplitude increases with the time-scale separation. The CV peak localizes at the points of maximal positive acceleration in the $I$-firing rate curve (a).

To understand the Monte Carlo data [Figs. 1(a)-1(c)], we note that the duty cycle shapes are uniform over $I$ so $H(t)$ is reset to a similar value every cycle. Hence, there is little correlation between subsequent periods and there are no mixed-mode oscillations. Suppose the duty cycle terminates at time $t=0$ and the AHP components are reset to their maximal values, and therefore decay as $H(t)=$ $\sum_{j} a_{j} e^{-t / \tau_{j}}$. Since the $H(t)$ dynamics is slow relative to (1), we can formulate the dynamical system as a stochastic escape from a slowly varying potential well $U(x, t)$, where $x$ represents the excitability state of the system. We derive $U(x, t)$ for the model (1) and (2) by approximating the dynamics by the normal form of the SNIC bifurcation

$$
\dot{x}=x^{2}-H(t)+I+\sigma \xi(t),
$$

where $U(x, t)=-x^{3} / 3+(I-H(t)) x$. For each time $t$ such that $H(t)-I=\Delta(t)>0$, where the system is below threshold for automatic firing, we calculate the mean first passage time [6] $T(\Delta(t))$ from the bottom of the well at the stable fixed point $x_{-}(t)=-\sqrt{\Delta(t)}$ of (3) to a point $x_{0}(t)=$ $\sqrt{\Delta(t)+1}$ that is beyond the noise dominated bottleneck in the vicinity of the unstable fixed point $x_{+}=\sqrt{\Delta(t)}$ of (3). Note that to compute $T(\Delta(t))$, the reflecting boundary at $x=-\infty$ is replaced by $x=-\sqrt{\Delta(t)+2}$ [7]. Following previous studies of molecular bond breaking [8], we assume adiabatic changes in $U(x, t)$ and approximate the probability of escape from the well during the interval $[t, t+d t)$ by $\lambda(\Delta(t)) P(t) d t$, where $\lambda(\Delta(t))=$ $T(\Delta(t))^{-1}$, and $P(t)$ is the proportion not escaped by time t. It follows that $\dot{P}(t)=-\lambda(\Delta(t)) P(t)$, so that $P(t)=$ $e^{-\int_{0}^{t} \lambda(\Delta(s)) d s} \Theta(\Delta(t))$, and the waiting time density is $p(t)=-\dot{P}(t)=\lambda(\Delta(t)) e^{-\int_{0}^{t} \lambda(\Delta(s)) d s} . \quad$ Thus, $\quad C V=$ $\sigma_{T} /\langle T\rangle$ with $\sigma_{T}^{2}=\left\langle T^{2}\right\rangle-\langle T\rangle^{2},\langle T\rangle=\int_{0}^{\infty} P(t) d t$, and $\left\langle T^{2}\right\rangle=\int_{0}^{\infty} t^{2} p(t) d t$, generating the results in Figs. 1(d)-1 (f), establishing that the stochastic escape formulation is qualitatively similar to the full system (1) and (2). We find that the adiabatic formulation is valid provided the AHP time scales are at least an order of magnitude larger than the time scale of (3).

The peaked $I-\mathrm{CV}$ relationship is robust to noise variation. Using the stochastic escape formulation, Fig. 2 shows $I$ vs mean frequency (a), and $I$ vs CV (b), over a range of noise values for the widely separated time-scale model 

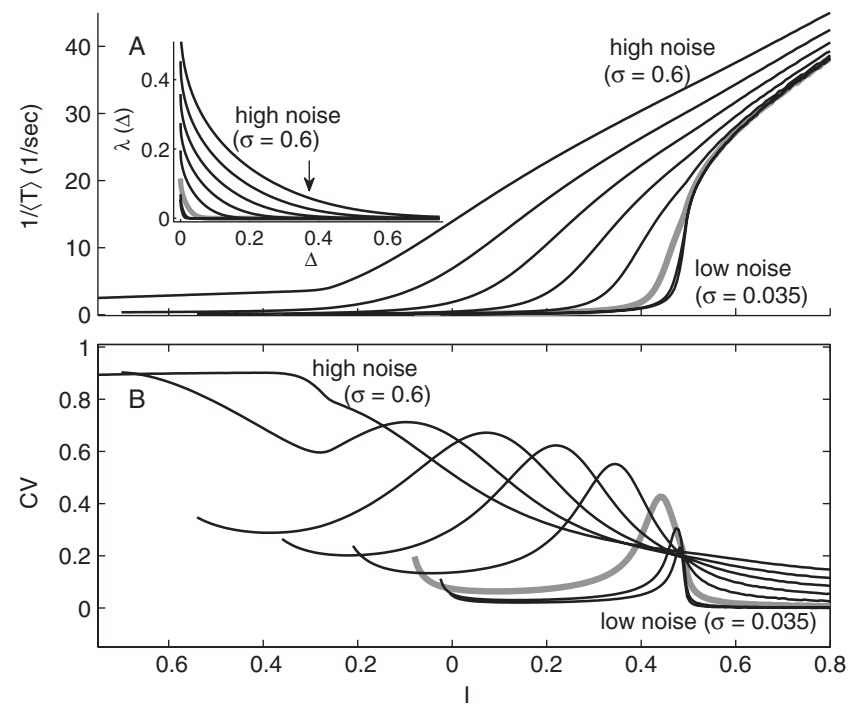

FIG. 2. Varying noise strength from $\sigma=0.035$ to 0.6 for the escape model with $\tau_{2}=5000$. The thicker gray line represents $\sigma=0.1$, the same as in Fig. 1(f). (a) Rate $1 /\langle T\rangle$ and $\lambda(\Delta)$ (inset), and (b) the CV.

shown in Fig. 1 ( $\tau_{2}=5000$, solid line). The thicker gray line represents the noise level used in Fig. $1(\sigma=0.1)$. The $\mathrm{CV}$ preserves the large peak over a wide range of noise levels but shifts to lower inputs and becomes broader. At high noise levels the peak "washes out" to a monotonic decreasing $I-C V$ relationship. The noise level that achieves this is very large $(\sigma=0.6)$, producing fluctuations nearly as large as the duty cycles in the full oscillator model (1) and (2). The inset in Fig. 2(a) shows $\lambda(\Delta)$ over the range of noise values, with the largest amplitude curve for the largest noise value $\sigma=0.6$, and smaller curves for the respective smaller noise values. Essentially, the broader tuning of $\lambda(\Delta)$ for large noise is less sensitive to the slow dynamics of $\Delta(t)$ and results in a "washout" of the CV peak. We note also that noise-dependent anticoherence resonance is observed in Fig. 2 for fixed $I$, similar to that found in [2].

The $I-C V$ phenomena in Figs. 1 and 2 are not unique to the oscillator model in (1) and (2). We have found similar peaked $I-C V$ relationships for multi-time-scale adaptation in an integrate-and-fire model, a Wilson-Cowan-type (SNIC) nonlinear oscillator, and a Hopf-type oscillator for both Monte Carlo and escape formulations (data not shown). All of these models can be described in terms of a stochastic escape problem involving an escape rate $\lambda(\Delta)$, and a slow, monotonically decaying function $\Delta(t)=$ $H(t)-I$. The phenomenon is relatively insensitive to a particular model choice because $\lambda(\Delta)$ for many standard models including (3) can be approximated well by a Kramer's rate [6] $\lambda(\Delta) \sim \beta \sigma^{2} \exp \left(-\alpha \Delta / \sigma^{2}\right)$, where $\alpha$, $\beta>0$ are parameters, with $\alpha$ relating $\Delta$ to the height of the potential well and $\beta$ accounting for the spike latency. The inset in Fig. 2(a) establishes this for the FitzHughNagumo-like model (3).
The CV peak for the two time-scale models in Figs. 1 and 2 is understood by noting that the shape of the $H(t)=$ $1.5 e^{-t / \tau_{1}}+0.5 e^{-t / \tau_{2}}$ is highly biphasic when $\tau_{2} \gg \tau_{1}$, possessing first a fast decaying phase followed by a slowly decaying phase. The peak of the CV occurs where the frequency-limiting component of the AHP mechanism transitions from the slow to the fast phase [e.g., $I \sim 0.45$ in Fig. 1(f)]. We note that no single-time-scale exponential has a sharp enough transition to produce a $\mathrm{CV}$ peak. However, a CV peak is observed for any $H(t)$ having a sharp transition and is not a special consequence of multiple-exponential decay. To show this we take a simple continuous piecewise-linear decay model $H(t)=$ $\left[\left(1-t / \tau_{1}\right) \Theta\left(t_{b}-t\right)+\left(\left(1-t_{b} / \tau_{1}\right)-\left(t-t_{b}\right) / \tau_{2}\right) \Theta(t-\right.$ $\left.\left.t_{b}\right)\right]^{+}$, where $t_{b}$ is the transition time between phases, the fast and slow phases are set by $\tau_{1}$ and $\tau_{2}$, respectively, $\Theta$ is the Heaviside function, and $[x]^{+}=x$ for $x>0$ and 0 for $x \leq 0$. Figures 3(a) and 3(b) show two such functions (thick gray lines): a more biphasic, wider time-scale separation in (a) $\left(\tau_{1}=7, \tau_{2}=55\right.$, in seconds), and a narrower time-scale separation in (b) $\left(\tau_{1}=12, \tau_{2}=40\right)$. The waiting time densities $p(t)$ (thin solid lines, rescaled to have unit height) computed with Kramer's approximation, are drawn on the same level as their associated $I$ values (horizontal dashed lines). The parameter choice for Kramer's rate $(\alpha=1, \beta=10, \sigma=0.1)$ resembles $\lambda(\Delta)$ thick line in Fig. 2(a) inset. We set $t_{b}$ so that $I=0.25=H\left(t_{b}\right)$ is the slow to fast transition for both models. Below threshold $[I<0$, rightmost $p(t)]$ the density climbs up then decays exponentially at a single rate $\lambda(-I)$, revealing Poisson-like escape after a delay. Above threshold $(I>0)$ all escape events precede the point of intersection $H(t)=I$ for in-
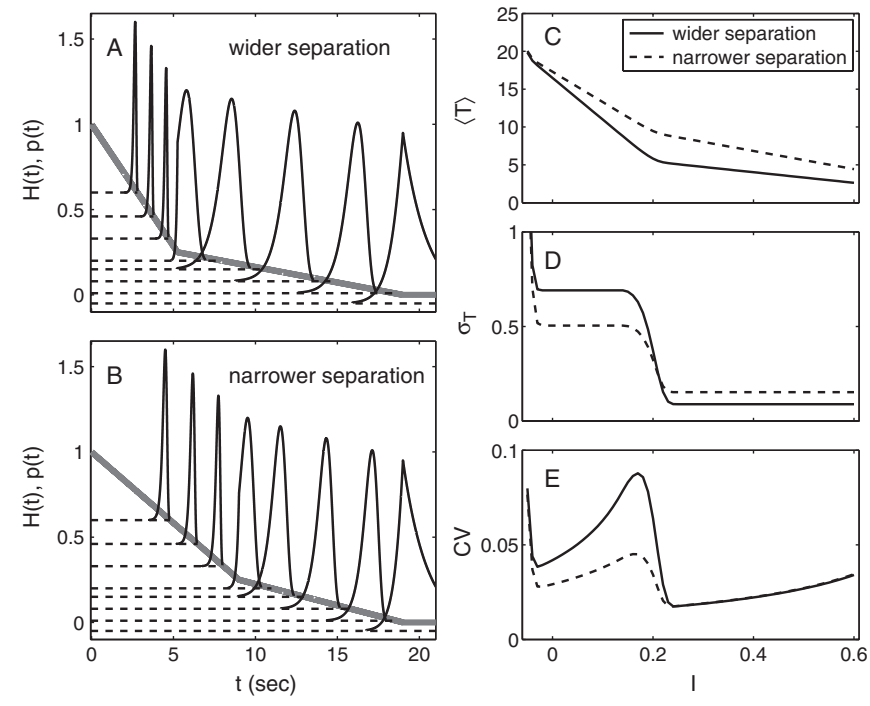

FIG. 3. Piecewise-linear AHP model. (a),(b) $H(t)$ (thick gray line) and $p(t)$ (thin solid lines) for wider time-scale separation and narrower time-scale separation, respectively. $p(t)$ is drawn on the level with its associated input: $I=-0.05,0.01,0.08$, $0.15,0.2,0.33,0.46,0.6$ (horizontal dashed lines). (c)-(e) period, $\mathrm{SD}$, and CV, respectively, for both $H(t) \mathrm{s}$. 


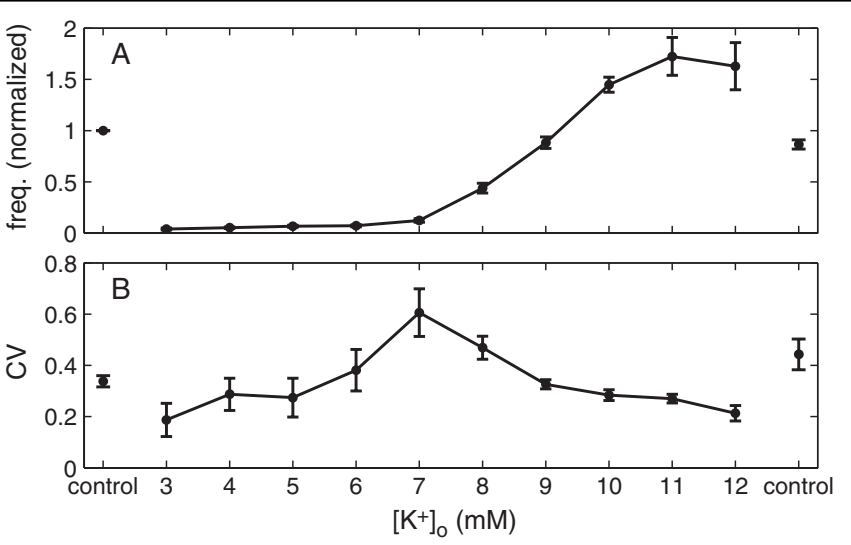

FIG. 4. (a) Frequency of the pre-BötC, normalized to $9 \mathrm{mM}$ control frequency, for inputs set by potassium concentration $\left[K^{+}\right]_{0} \quad(\mathrm{mM}) \quad(n=13$, standard error bars indicated $)$, and (b) CV. Intermediate excitability levels produced a peaked CV, similar to the above results.

stantaneous firing. For inputs intersecting sufficiently below the transition point $I=0.25$, the associated densities have a similar shape, but are translated on the $t$ axis proportional to $I$, producing a linear decline in average periods (c), but constant $\sigma_{T}$ (d). Here, $\sigma_{T}$ is constant because the rate of approach to threshold $[\Delta(t) \rightarrow 0]$ is the same for a range of $I$ s intersecting $H(t)$ on the slow phase. This produces an increasing CV (e). When $I \sim 0.25$, where the system transitions from the slow to the fast phase, the periods decrease at a lower linear rate and $\sigma_{T}$ drops dramatically to a lower but constant value, reflecting a different rate of approach to threshold for $\Delta(t)$ on the fast phase of $H(t)$. This transition through $I \sim 0.25$ produces the peak in the $\mathrm{CV}$ as $\sigma_{T}$ drops. For the wider separated time-scale model [solid line in (c)-(e)] this transition is more substantial than the less separated time-scale model [dashed line in (c)-(e)], producing a larger $\mathrm{CV}$ peak. Beyond $I=0.25$ the $\mathrm{CV}$ starts to increase again, due to constant $\sigma_{T}$ and decreasing $\langle T\rangle$.

The peaked CV of a noise-driven system with biphasic adaptation provides an explanation for input-controlled oscillation regularity in a slice preparation containing the pre-BötC. The in vitro oscillation periods can range from 2-3 min down to $2 \mathrm{sec}$ in neonatal mice, controlled by excitability set by bath concentration of potassium $\left[\mathrm{K}^{+}\right]_{0}$. Figure 4 shows the frequency (a) and CV (b) of oscillations, measured from XII cranial nerve, over a range of $\left[K^{+}\right]_{0}$ for $n=13$ slices. For each slice the frequency was first measured at $9 \mathrm{mM}$ control (frequency: $0.20 \pm 0.02 \mathrm{~Hz}$ and period: $5.7 \pm 0.51 \mathrm{~s}$ ) then washed successively from $3 \mathrm{mM}$ producing very slow rhythms (frequency: $0.008 \pm$ $0002 \mathrm{~Hz}$ and period: $143.2 \pm 3.9 \mathrm{~s}$ ), up to $12 \mathrm{mM}$ producing the peak frequency at 11 or $12 \mathrm{mM}$ : $0.29 \pm 0.02 \mathrm{~Hz}$ (period: $3.5 \pm 0.2 \mathrm{~s}$ ) and normalized to the control frequency. After $12 \mathrm{mM}$, each slice was brought back to
$9 \mathrm{mM}$ for consistency. The peaked $\mathrm{CV}$ for intermediate $\left[\mathrm{K}^{+}\right]_{0}$ levels located at the point of maximal acceleration in the rate curve is evident. According to our theory, the wide range of periods and the peaked $\mathrm{CV}$ is due to a widely separated multi-time-scale adaptation. Since little is known about the particular adaptation mechanisms in the preBötC slice [9], this provides an important experimental prediction.

In conclusion, our work demonstrates that noise-driven oscillatory systems with highly separated multiple timescale adaptation, exhibiting biphasic fast-then-slow decay can produce nonmonotonic relationships between oscillation regularity $(\mathrm{CV})$ and excitability, exhibiting a peak of maximal oscillation incoherence for intermediate inputs. Such a process may account for experimental observations regarding oscillation regularity in breathinglike rhythms of the pre-BötC. We note similar phenomena exist with more than two time scales provided the time-scale components cluster in two separated aggregates. Furthermore, doublepeaked input-CV profiles can be observed with separated three-time-scale aggregates (data not shown). The mechanism underlying such input-dependent oscillation regularity is distinct from noise-dependent coherence and anticoherence resonance found in single-time-scale adaptation systems, which only exhibit monotonic decreasing I-CV relationships [2]. Moreover, the formulation of the phenomenon as a stochastic escape problem shows it to be generally applicable to any noisy oscillator for which threshold crossing can be described in terms of escape from a potential well whose height decreases slowly and biphasically. Analogous results may also apply to molecular bond breaking for appropriately chosen time-dependent forcing.

WHN is supported by RTG 0354259, and NSF DGE0217424; CADN by NSF IOB 0616099; and PCB by NSF DMS0515725.

[1] A. Pikovsky and J. Kurths, Phys. Rev. Lett. 78, 775 (1997).

[2] B. Lindner, L. Schimansky-Geier, and A. Longtin, Phys. Rev. E 66, 031916 (2002).

[3] B. Hille, Ion Channels of Excitable Membranes (Sinauer, Sunderland, MA, 2001), 3rd ed.

[4] J. C. Smith, H. H. Ellenberger, K. Ballanyi, D. W. Richter, and J. L. Feldman, Science 254, 726 (1991).

[5] W. H. Nesse, A. Borisyuk, and P. C. Bressloff, J. Comput. Neurosci. (to be published).

[6] C.W. Gardiner, Handbook of Stochastic Methods (Springer, New York, 2004), 3rd ed.

[7] B. Lindner and A. Longtin, Neural Comput. 15, 1761 (2003).

[8] O. K. Dudko, G. Hummer, and A. Szabo, Phys. Rev. Lett. 96, 108101 (2006).

[9] C. A. Del Negro, K. Kam, J. A. Hayes, and J. L. Feldman (to be published). 